\title{
Traffic Improvements in Wireless Communication Networks Using Antenna Arrays
}

\author{
Javad Razavilar, Member, IEEE, Farrokh Rashid-Farrokhi, Member, IEEE, and K. J. Ray Liu, Senior Member, IEEE
}

\begin{abstract}
A wireless network with beamforming capabilities at the receiver is considered that allows two or more transmitters to share the same channel to communicate with the base station. A novel approach is introduced, which combines the effects of the digital signal processing (adaptive beamforming) at the physical layer withthe traffic policies at the network layer on the overall queuing model of a cell. The effect of signal processing on the queuing model of the cell is represented by a parameter in the final cell model. Each cell is modeled by a multiuser/multiserver service facility, where each server is a beamformed channel formed by the cell's base station. From this effective cell model, we find the closed form solutions for blocking probabilities of the calls and total carried traffic in a wireless netwrok with adaptive arrays. Our analytical as well as numerical results show that adaptive beamforming at the receiver reduces the blocking probability of the calls and increases the total carried traffic in the system.
\end{abstract}

Index Terms-Adaptive beamforming, antenna arrays, handoff queuing, wireless networks.

\section{INTRODUCTION}

$\mathbf{I}$ $\mathrm{N}$ A cellular wireless communication network, as a mobile user crosses the boundaries of cells (coverage area of each base station) the call must be handed over to the new base station (BS) that provides the best link quality. This operation is called a handoff and ideally goes unnoticed by the customer [1], [2]. An important problem arising from the mobility of the customers in a cellular system is the possible blocking of a handoff call [3]-[5]. Namely, we can have a call that was initiated in one cell in the system and moves into a new cell where all the channels (servers) happen to be already occupied by other calls. This entering call will then have to be cleared from the system involuntarily, which is obviously an unpleasant situation for the customer, and it is called forced termination of a call. Such an event results in a disconnection in the middle of a call, which is highly undesirable.

Due to fixed finite resources, we cannot assume that more physical radio channels will be added to a spectrum allocation to reduce the probability of blocking or forced termination [1]. The reallocation of bandwidth is a standards-setting process that takes a considerably long time. The increasing popularity

Manuscript received September 15, 1998; revised August 31, 1999

J. Razavilar is with the Technology Development Center, 3COM Corporation, San Diego, CA 92128 USA (e-mail: javad_razavilar@3com.com).

F. Rashid-Farrokhi is with the Wireless Communications Research Department, Bell Labs, Lucent Technologies, Holmdel, NJ 07733 USA (e-mail: farrokhi@lucent.com).

K. J. R. Liu is with the Electrical Engineering Department and Institute for Systems Research, University of Maryland, College Park, MD 20742 USA (e-mail: kjrliu@eng.umd.edu).

Publisher Item Identifier S 0733-8716(00)01293-2. of wireless communication services together with the limited amount of the available radio spectrum calls for highly efficient usage of wireless network resources, including traffic channels [6], [7].

The interference reduction capability of antenna arrays have been considered as a means to increase the capacity of wireless systems [8]-[18]. Specifically, using beamforming techniques at the receiver, two or more transmitters can share the same traffic channel to communicate with the base station at the same time. An adaptive antenna array is used at the base station to form several antenna beams simultaneously. Each beam captures one transmitter by automatically pointing its pattern toward that transmitter while nulling other cochannel transmitters [8], [15]-[17]. In this way, the cochannel interference is minimized, and therefore the signal-to-(interference plus noise) ratio (SINR) for the signal of interest is maximized.

In urban wireless environments, the transmitted signal by each user is reflected by surrounding buildings and the terrain. Therefore several copies of the transmitted signal are received at the base station with different delays and different attenuation associated with each path. This effect is called multipath fading. It is assumed that for each transmitter there are at most $\alpha$ strong multipath components received at the base station. An adaptive array with $M$ antenna elements forms up to $M-1$ array nulls. In this paper we only consider space-only diversity combining. In our analysis, we assume that there are enough array nulls available to reject the interference caused by cochannel transmitters as well as multipath interference. In a space-time diversity combining, spatial nulls can be used to reject the cochannel interference (CCI), while intersymbol interference (ISI) can be eliminated by time diversity.

This paper considers a wireless network with beamforming capabilities at the receiver that allows two or more transmitters share the same channel to communicate with the base station. Each cell is modeled by a multiuser multiserver service facility. Each server is a beam-formed channel formed by the cell's base station. For a set of cochannel transmitters the probability of successful capture by a separate antenna beam is computed. The success probabilities are taken into account in the queuing model of the system. From this generalized model the closed form blocking probabilities of the calls and total carried traffic in the system under different traffic policies are derived.

This paper is organized as follows. A review of spatial multiplexing using adaptive beamforming techniques forms the subject of Section II. A network with omnidirectional antenna and guard channel traffic policy is presented in Section III. A network model with joint beamforming and handoff using antenna arrays and the analysis of blocking probabilities under 


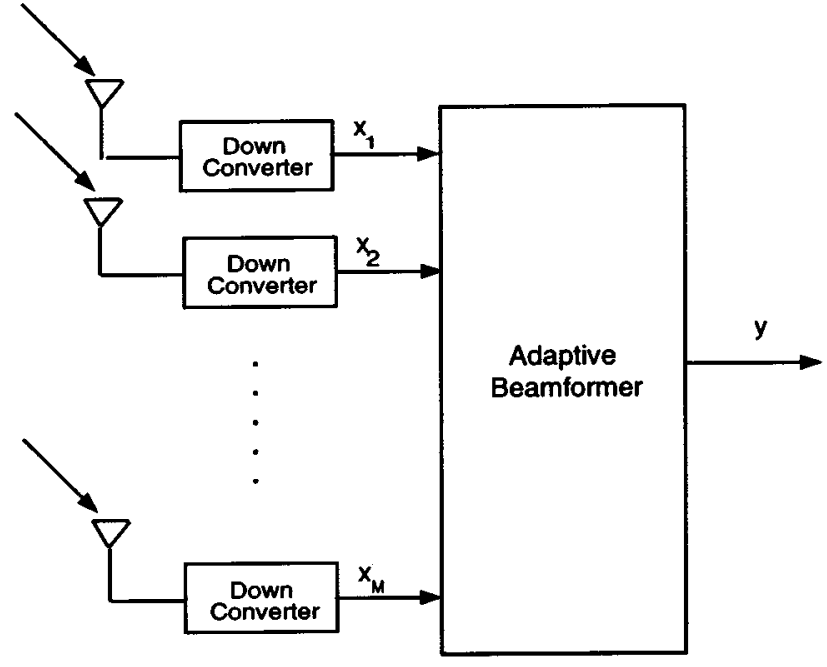

Fig. 1. A diversity combining system.

guard channel policy are presented in Section IV. Analysis of the network with antenna arrays at the receiver under queuing of handoff calls traffic policy, for an infinite as well finite queue lengths, are discussed in Section V. Numerical results are presented in Section VI. Section VII includes our conclusions and remarks.

\section{Review of Spatial Multiplexing Using Adaptive BEAMFORMING}

An antenna array is a multibeam adaptive array with its gain pattern being adjusted dynamically [9]. For a fixed $M$ beam sectorized antenna and an $M$ element antenna array system the antenna gain and system capacity are increased $M$-fold. Nevertheless, the difference is that in a fixed beam system the handoff from one beam to another is the main disadvantage, while in an antenna array system each beam will track its user within the coverage of a cell. Moreover, antenna array systems provide diversity gain against multipath signals and strong interferers by placing antenna nulls toward those sources.

Space and time diversity are used as a means to reduce the $\mathrm{CCI}$ and ISI. If the desired signal and the cochannel signals have different temporal or spatial signatures, using time and space diversity can improve the signal-to-noise ratio (SNR). The block diagram of an adaptive array is depicted in Fig. 1.

In general, the objective is to form the main beam toward the desired user and nulls toward the interference sources as illustrated in Fig. 2.

We shall use adaptive beamforming capabilities of antenna arrays to maintain a constant gain for the signal along the direction of interest and adjusting the nulls so as to reject the cochannel interference. In this way the interference is minimized and SINR for the signal of interest is maximized. A single base station equipped with an $M$ element adaptive array is considered. Assume there are $J$ cochannel users in the cell. Let $s_{j}(k)$ for $j=1,2, \ldots, J$ denote the $j$ th transmitted signal. We assume that at most $N$ multipath signals from each user would

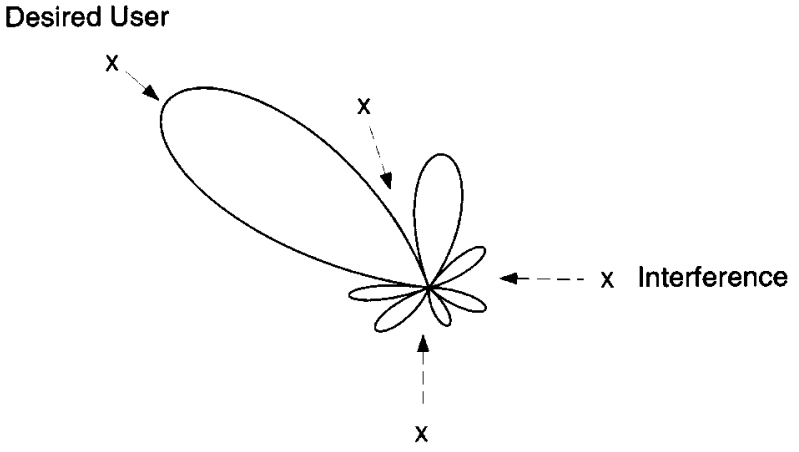

Fig. 2. Sample array pattern.

arrive in the base station with different delays $\left(\tau_{n}\right)$. Therefore the received signal vector would be

$$
\mathbf{x}(k)=\sum_{j=1}^{J} \sqrt{P_{j} G_{j}} \sum_{n=1}^{N} \alpha_{j}^{n} \mathbf{a}\left(\theta_{j}^{n}\right) s_{j}\left(t-\tau_{n}\right)+\mathbf{n}(k)
$$

where $\theta_{i}^{n}$ is the arrival direction of the $n$th multipath signal from the $i$ th user, $P_{i}$ is the power of the $i$ th transmitter, $G_{i}$ is the link gain between the $i$ th transmitter and the base station, $\alpha_{i}^{n}$ is the $n$th path fading, $\mathbf{n}(k)$ is a zero mean Gaussian random vector with variance of each element $\sigma^{2}$, and $\mathbf{a}\left(\theta_{i}^{n}\right)$ is the array response to the multipath signal arriving from direction $\theta_{i}^{n}$ with

$$
\mathbf{a}\left(\theta_{i}^{n}\right)=\left[a_{1}\left(\theta_{i}^{n}\right), a_{2}\left(\theta_{i}^{n}\right), \ldots, a_{M}\left(\theta_{i}^{n}\right)\right]^{T} .
$$

\section{A. Space Diversity Combining}

In a system with adaptive arrays, a weighted sum of the received signals is combined at the output of beamformer as illustrated in Fig. 3

$$
y_{i}(k)=\mathbf{w}_{i}^{H} \mathbf{x}(k)
$$

where $i$ denotes the index for the signal of interest, $\mathbf{w}_{i}^{T}=\left[w_{i 1}, \ldots, w_{\mathrm{iM}}\right]$ is the weight vector, and $\mathbf{x}^{T}(k)=$ $\left[x_{1}(k), \ldots, x_{M}(k)\right]$ is the received signal vector sampled at the output of the down-converters.

\section{B. Minimum Variance Distortionless Response (MVDR)}

We can choose the weight vector $\mathbf{w}_{i}$ to steer a beam toward the direction of signal of interest $\theta_{i}$ and adjust the nulls to reject the interference. This is done by maintaining a distortionless response in the direction of interest and placing the nulls in the directions of other cochannel interferers. The average output power is given by

$$
E_{i}=E\left[y_{i}(k) y_{i}^{H}(k)\right]=\mathbf{w}_{i}^{H} E\left[\mathbf{x}(k) \mathbf{x}^{H}(k)\right] \mathbf{w}_{i}=\mathbf{w}_{i}^{H} \boldsymbol{\Phi}_{i} \mathbf{w}_{i}
$$

with

$$
\begin{aligned}
\boldsymbol{\Phi}_{i}=P_{i} G_{i} \alpha_{i}^{1} \mathbf{a}_{i}\left(\theta_{i}^{1}\right) \mathbf{a}_{i}^{H}\left(\theta_{i}^{1}\right) \\
+\sum_{j=1, j \neq i}^{J} P_{j} G_{j} \sum_{n=2}^{N} \alpha_{j}^{n} \mathbf{a}_{j}\left(\theta_{j}^{n}\right) \mathbf{a}_{j}^{H}\left(\theta_{j}^{n}\right)+\frac{N_{0}}{2} I
\end{aligned}
$$




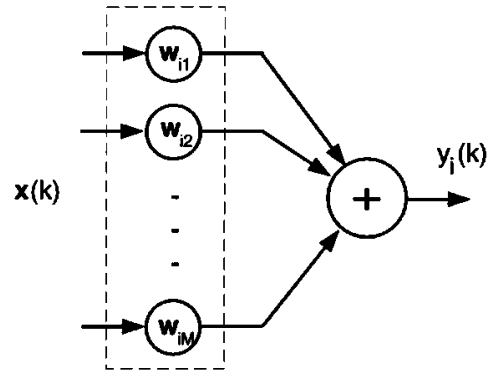

Fig. 3. Space diversity combiner.

The correlation matrix due to the interference terms is defined as

$$
\boldsymbol{\Phi}_{i}^{N}=\sum_{j=1, j \neq i}^{J} P_{j} G_{j} \sum_{n=2}^{N} \alpha_{j}^{n} \mathbf{a}_{j}\left(\theta_{j}^{n}\right) \mathbf{a}_{j}^{H}\left(\theta_{j}^{n}\right)+\frac{N_{0}}{2} I .
$$

As was pointed out before, if the array response to the direction of the desired user is known, the beamformer tries to minimize the variance of the beamformer output subject to maintaining a distortionless response in the direction of interest such that $\mathbf{w}_{i}^{H} \mathbf{a}_{i}\left(\theta_{i}^{1}\right)=1$. For this reason, this adaptive beamformer is called an MVDR [11] beamformer. Now from (4) and (5) along with the constraint that $\mathbf{w}_{i}^{H} \mathbf{a}_{i}\left(\theta_{i}^{1}\right)=1$, the received signal power plus the interference power as a function of $\mathbf{w}_{i}$ is

$$
\begin{aligned}
& E_{i}=P_{i} G_{i}+\sum_{j=1, j \neq i}^{J} P_{j} G_{j} \sum_{n=2}^{N} \alpha_{j}^{n} \mathbf{w}_{i}^{H} \mathbf{a}_{j}\left(\theta_{j}^{n}\right) \\
& \cdot \mathbf{a}_{j}^{H}\left(\theta_{j}^{n}\right) \mathbf{w}_{i}+\frac{N_{0}}{2} \mathbf{w}_{i}^{H} \mathbf{w}_{i} .
\end{aligned}
$$

Let $I_{i}$ denote the total interference plus noise power given by

$I_{i}=\sum_{j=1, j \neq i}^{J} P_{j} G_{j} \sum_{n=2}^{N} \alpha_{j}^{n} \mathbf{w}_{i}^{H} \mathbf{a}_{j}\left(\theta_{j}^{n}\right) \mathbf{a}_{j}^{H}\left(\theta_{j}^{n}\right) \mathbf{w}_{i}+\frac{N_{0}}{2} \mathbf{w}_{i}^{H} \mathbf{w}_{i}$.

In (7), $P_{i} G_{i}$ is the received power from the signal of interest while $I_{i}$ given by (8) is the contribution to the output power $E_{i}$ from the interference and noise. The optimum weight vector $\hat{w}$ for the MVDR problem is given by

$$
\hat{\mathbf{w}}_{i}=\frac{\Phi^{-1} \mathbf{a}_{i}\left(\theta_{i}^{1}\right)}{\mathbf{a}_{i}^{H}\left(\theta_{i}^{1}\right) \Phi^{-1} \mathbf{a}_{i}\left(\theta_{i}^{1}\right)} .
$$

Since the desired signal (arriving along $\theta_{i}$ ) will not be affected by the beamforming process and only the interference is rejected, the SINR is maximized for the signal in the direction of interest, i.e.,

$\Gamma_{i, \max }=$

$\frac{P_{i} G_{i}}{\sum_{j=1, j \neq i}^{J} P_{j} G_{j} \sum_{n=2}^{N} \alpha_{j}^{n} \hat{\mathbf{w}}_{i}^{H} \mathbf{a}_{j}\left(\theta_{j}^{n}\right) \mathbf{a}_{j}^{H}\left(\theta_{j}^{n}\right) \hat{\mathbf{w}}_{i}+\frac{N_{0}}{2} \hat{\mathbf{w}}_{i}^{H} \hat{\mathbf{w}}_{i}}$

\section{Minimum Mean-Square Error (mMSE)}

If the array response is not known, we can employ a training sequence and minimize the difference between the training se- quence and the output of the beamformer in mean square sense, that is

$$
\hat{\mathbf{w}}_{i}=\arg \min _{w_{i}} E\left[\left|d_{i}-\mathbf{w}_{i}^{H} \mathbf{a}_{i}\left(\theta_{i}^{1}\right)\right|^{2}\right] .
$$

The solution to this problem is given by [11]

$$
\hat{\mathbf{w}}_{i}=\Phi_{i}^{-1} \mathbf{p}_{i}
$$

where $\Phi_{i}$ is defined as before and $\mathbf{p}_{i}$ is the cross correlation between the received vector and the training sequence; $\mathbf{p}_{i}=$ $E\left[\mathrm{x}_{i} d_{i}^{*}\right]$. The maximum CIR in this case is given by

$$
\begin{aligned}
& \Gamma_{i, \max }= \\
& \sum_{j=1, j \neq i}^{J} P_{j} G_{j} \sum_{n=2}^{N} \alpha_{j}^{n} \hat{\mathbf{w}}_{i}^{H} \mathbf{a}_{j}\left(\theta_{j}^{n}\right) \mathbf{a}_{j}^{H}\left(\theta_{j}^{n}\right) \hat{\mathbf{w}}_{i}+\frac{N_{0}}{2} \hat{\mathbf{w}}_{i}^{H} \hat{\mathbf{w}}_{i}
\end{aligned}
$$

\section{NETWORK WITH OMNIDIRECTIONAL ANTENNA AND GuARd ChanNEls TRAFFIC POLICY}

In this section we review the traffic policies proposed in [4] and [5] that can help to decrease the blocking probability of handoff calls, while not penalizing the originating calls too much. In this paper, the following assumptions are made for the network model.

- We regard a cell as a service facility with, say, $L$ physical channels. Two types of traffic are handled by the cell: the handoff calls and the originating calls. Once a call is in progress, its original type (handoff or originating) cannot be distinguished.

- Poisson (memoryless) arrivals for originating calls with rate equal to $\gamma$ per second (i.e., originating calls have an exponential interarrival time distribution with parameter $\gamma)$

- Poisson arrivals for handoff calls with rate equal to $\lambda$ per second (i.e., handoff calls have an exponential interarrival time distribution, with parameter $\lambda$ ).

- Exponential (memoryless) service time, on the average equal to $1 / \eta \mathrm{s}$. The service time refers to the entire call duration, assumed independent of the number of handoffs to other cells.

- Exponential handoff service rate with rate equal to $\nu$ per second per call (i.e., the average duration between two consecutive handoffs is $1 / \nu$ ).

The guard channels traffic policy is the blocking of newly originating calls as soon as $g$ channels among the initial $L$ channels are still free. This allows us to build a guard band before handoff calls will be blocked, which will happen only when all $L$ channels are occupied. In what follows, this policy is quantified in order to illustrate the usefulness of the guard channel concept. First we define some useful notations

$$
\alpha=\lambda+\gamma, \quad \mu=\eta+\nu, \quad a=\frac{\alpha}{\mu}, \quad b=\frac{\gamma}{\mu}, \quad c=\frac{\lambda}{\mu} .
$$

We recall that $\mu$ is the total service rate, $\gamma$ is the arrival rate of originating calls, and $\lambda$ is the arrival rate of the handoff calls. So $a$ can be considered as the total traffic offered to the cell, and $c$ 
is the handoff traffic offered to the cell. $g$ is the number of guard channels, and it is desired to determine an appropriate value of $g$ in order to protect handoff calls from high blocking without increasing the blocking of originating calls too much [4], [5]. This system is a simple blocking system with memoryless arrivals and memoryless service time, as well as state-dependent arrival rate. Namely, the arrival rate into the cell is equal to $(\lambda+\gamma)$ if we have fewer than $L-g$ customers present in the system, and simply equal to $\lambda$ (corresponding to handoff calls) if we have $L-g$ or more customers in the system. The number of users in the system $n$ for $n=0,1, \ldots, L$ forms a discrete Markov chain with the state transition diagram shown in Fig. 4.

For the state probabilities, $P(n) n=0,1, \ldots, L$, we have

$$
\begin{cases}0 \leq n \leq L-g & P(n)=\frac{a^{n}}{n !} P(0) \\ L-g \leq n \leq L & P(n)=\frac{a^{L-g} c^{n-L-g)}}{n !} P(0)\end{cases}
$$

where

$$
P(0)=1 /\left[\sum_{n=0}^{L-g-1} \frac{a^{n}}{n !}+a^{L-g} \sum_{n=L-g}^{L} \frac{c^{n-(L-g)}}{n !}\right] .
$$

The blocking probability $B_{O}$ of originating calls is then given by

$$
\begin{aligned}
B_{O} & =P[n \geq L-g] \\
\Rightarrow B_{O} & =\left[a^{L-g} \sum_{n=L-g}^{L} \frac{c^{n-(L-g)}}{n !}\right] P(0) .
\end{aligned}
$$

Similarly for the handoff calls, we have the blocking probability $B_{H}$ given by

$$
\begin{aligned}
B_{H} & =P[n=L] \\
\Rightarrow B_{H} & =\frac{a^{L-g_{C} g}}{L !} P(0) .
\end{aligned}
$$

\section{NETWORK WITH ADAPTIVE ARRAY AND GUARD CHANNELS TRAFFIC POLICY}

In this section we analyze the network with adaptive array under guard channels traffic policy [5]. Fig. 6 illustrates our proposed channel assignment policy. From Fig. 6, it is clear that first $L$ users are assigned to $L$ physical channels. If all $L$ physical channels are busy, the new call will be served by one of the beam-formed channels, using the following algorithm. It is assumed that upon arrival of the $(L+1)$ th call, the adaptive array points one beam toward that user, and one beam-formed channel (associated with one of the $L$ physical channels) will be assigned to that call. Each of the physical channels can serve up to $K$ different calls by $K$ separate beams. This is feasible by deploying $K$ beamformers in parallel for each channel, as shown in Fig. 5. In this setup, a maximum of $K L$ calls can be served in the cell.

Let us assume that $(i-1)$ cochannel transmitters successfully share the same channel. The newly arrived $i$ th transmitter is allowed to share that channel with other cochannel calls if $\Gamma_{i} \geq \gamma$, where $\Gamma_{i}$ is given by (10) and the threshold $\gamma$ is a system parameter determined by the governing standard. For instance, in the $I S$-54 standard, $\gamma$ is $14 \mathrm{~dB}$, while in AMPS it is $18 \mathrm{~dB}$.

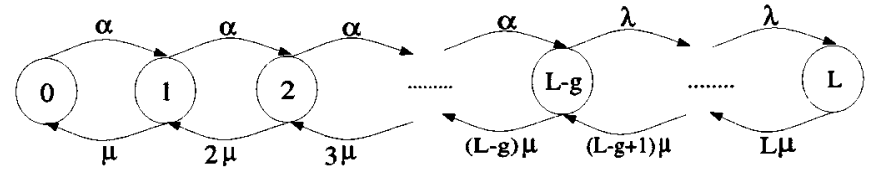

Fig. 4. State transition diagram of discrete Markov chain, the network model of the system with guard channels.

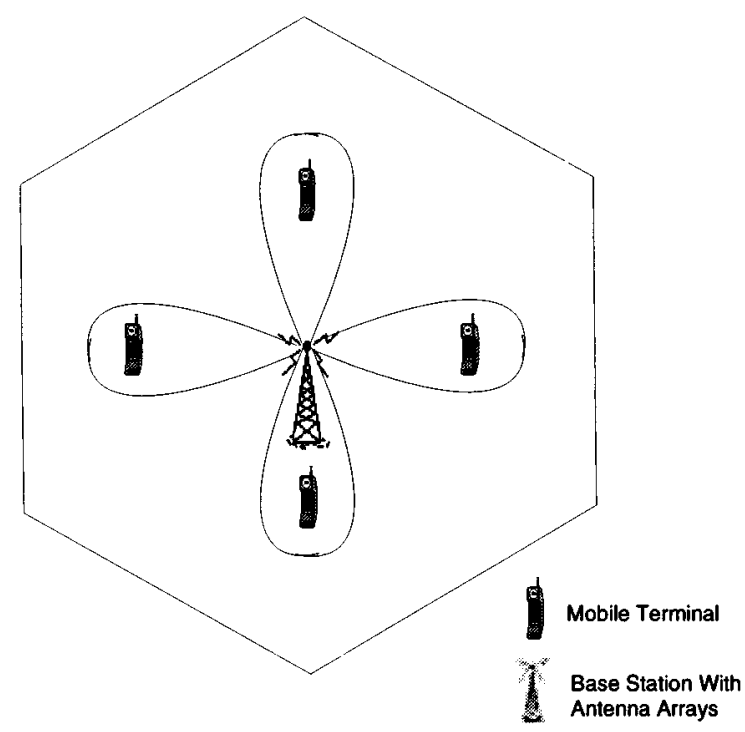

Fig. 5. Capturing four mobile users over the same channel successfully by an adaptive antenna array at the BS.

To avoid degradations, a call admission control (CAC) mechanism is devised. If $\Gamma_{i}<\gamma$ or $i>K$, that user is not accepted into the system. The probability of the event $\Gamma_{i} \geq \gamma$ in the system would yield the success probability $p_{i \mid i-1, M}$ that the $i$ th transmitter can share the same channel, given that $i-1$ transmitters are already using that channel. Clearly the success probability $p_{i \mid i-1, M}$ depends on $M$ and determined by the following parameters: distribution model of the users over the coverage area of the base station, path loss model of the system, which determines the link gains; $G_{i}$ 's, threshold $\gamma$, and SNR. In this paper, we will assume that the mobile terminals are uniformly distributed around the base station over the circular plane with normalized radius 1 . For the path loss, we assume that the level of a terminal at the base station will be proportional to $1 / x^{\beta}$, where $x$ is the distance between the terminal and the base station. The exponent $\beta$ will be a number between two and about five $(\beta=2-5)$.

Considering the complex probabilistic structure of the problem due to beamforming process, it is very hard, if not impossible, to compute the success probabilities directly. For this reason, we resort to Monte Carlo simulations to obtain the success probabilities. Success probabilities close to one are expected for high SNR. On the other hand, for low to medium SNR's, the success probabilities are expected to increase as the number of antenna elements $M$ increases or threshold $\gamma$ decreases. Our simulation results confirms the above heuristic results. Figs. 7 and 8 illustrate Monte Carlo simulation results for the success probabilities $p_{2 \mid 1, M}$ and $p_{3 \mid 2, M}$, for two- and three-beam adaptive arrays respectively, where $M$ denotes the number of antenna elements. It is worth mentioning that the 


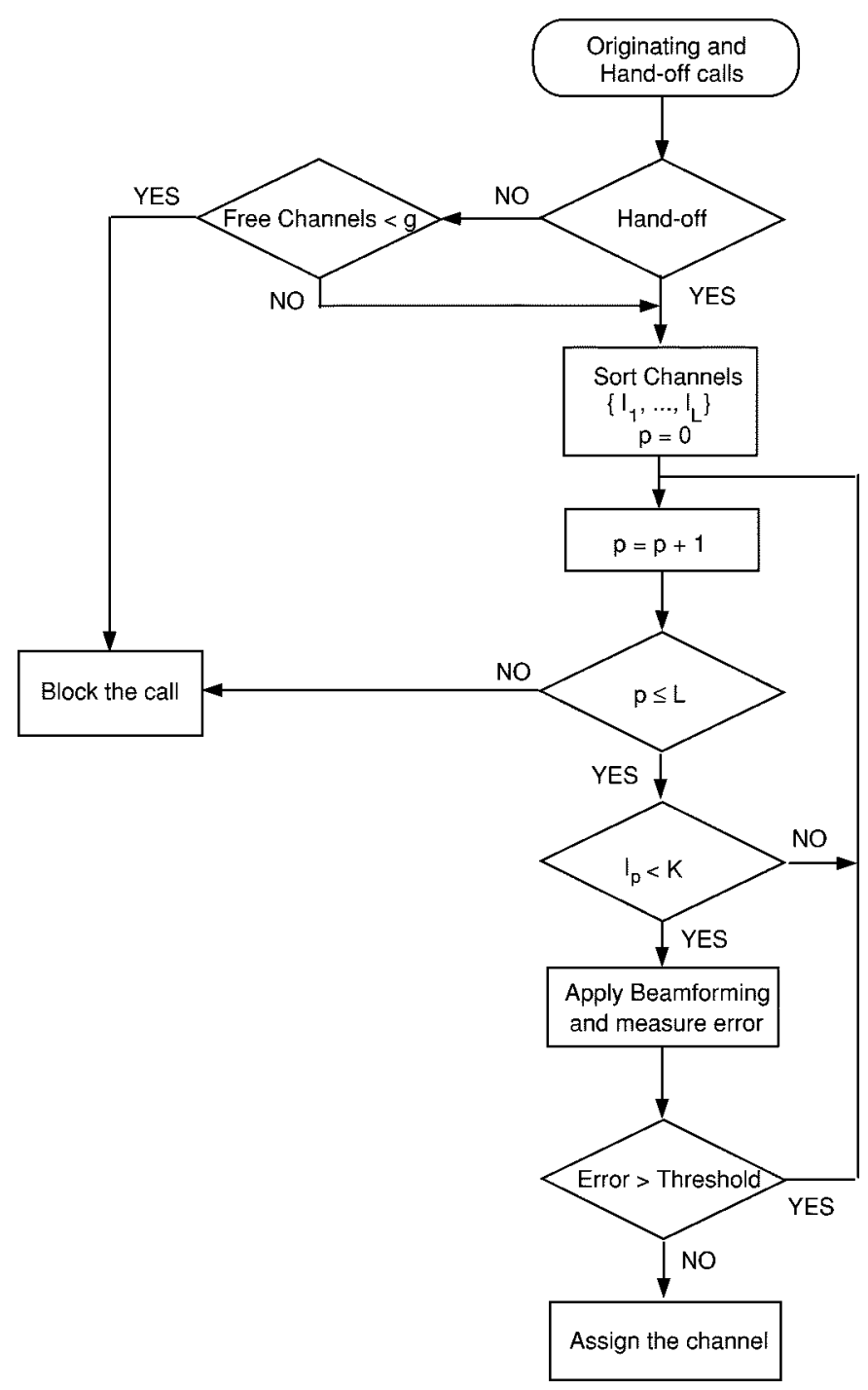

Fig. 6. Flowchart of the channel assignment algorithm in a system with antenna array.

success probabilities obtained through simulations are valid for uplink as well as downlink. However, in this paper, the success probabilities are used for deriving the overall queuing model at the base station. Therefore, they are used for uplink analysis only.

Now let us compute the probability of successful admission to the system, using $p_{i \mid i-1, M}$. Since there are $L$ distinct channels in the system and each channel may be reused up to $K$ times, we define the probability of successful admission of the $(n+1)$ th user into the system given that there are already $n$ users in the system as

$$
q_{n+1 \mid n}=q_{N_{t+1} \mid N_{t}}\left(N_{t+1}=n+1 \mid N_{t}=n\right)
$$

where $N_{t}$ is the number of users in the system at time $t$ before a new call (user) arrives into the system and $t$ is the time index, which increases by one at each epoch corresponding to a new handoff or originating call. For a $K$-beam adaptive array system, let $\left(x_{2}, x_{3}, \ldots, x_{K}\right)$ denote the beamformed channels occupancy vector, where $x_{k}$ denotes the number of physical channels, which have at least $k$ beams, and $0 \leq x_{k} \leq L$

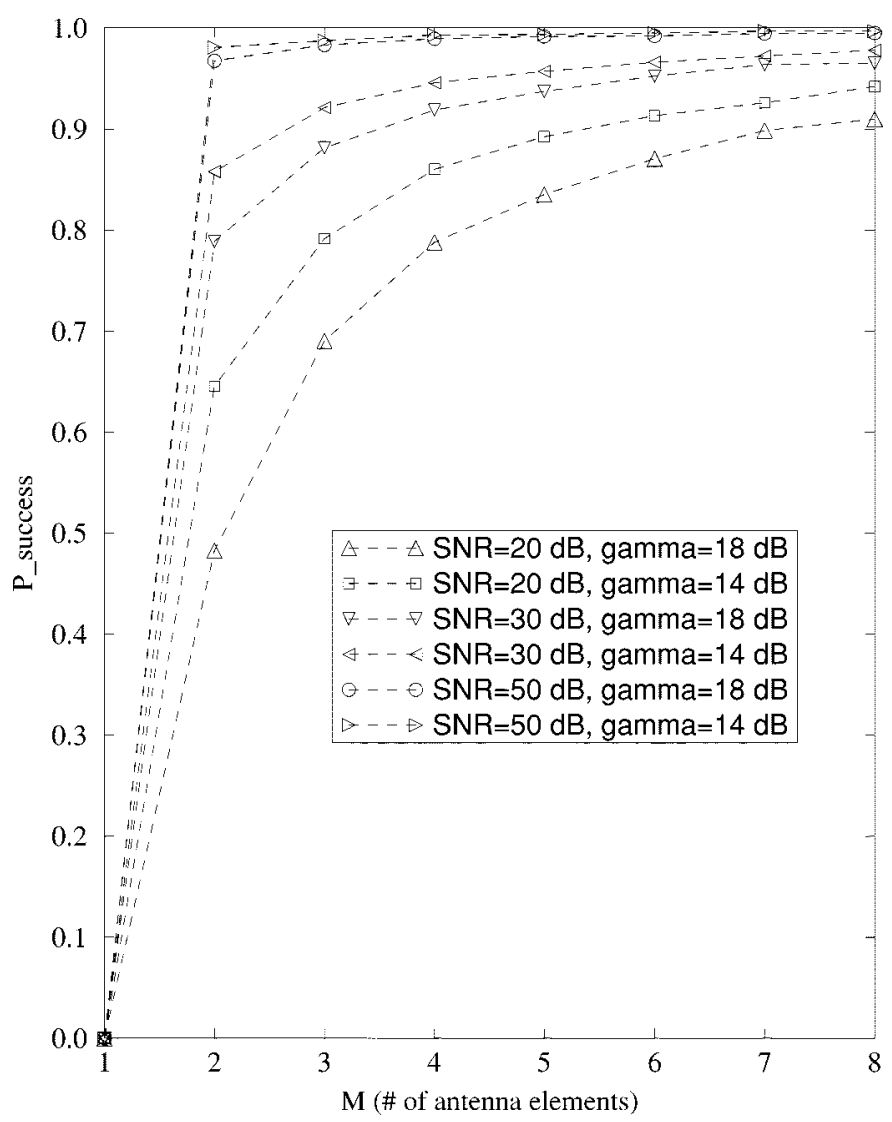

Fig. 7. Success probability $p_{2 \mid 1, M}$ for a two-beam adaptive array.

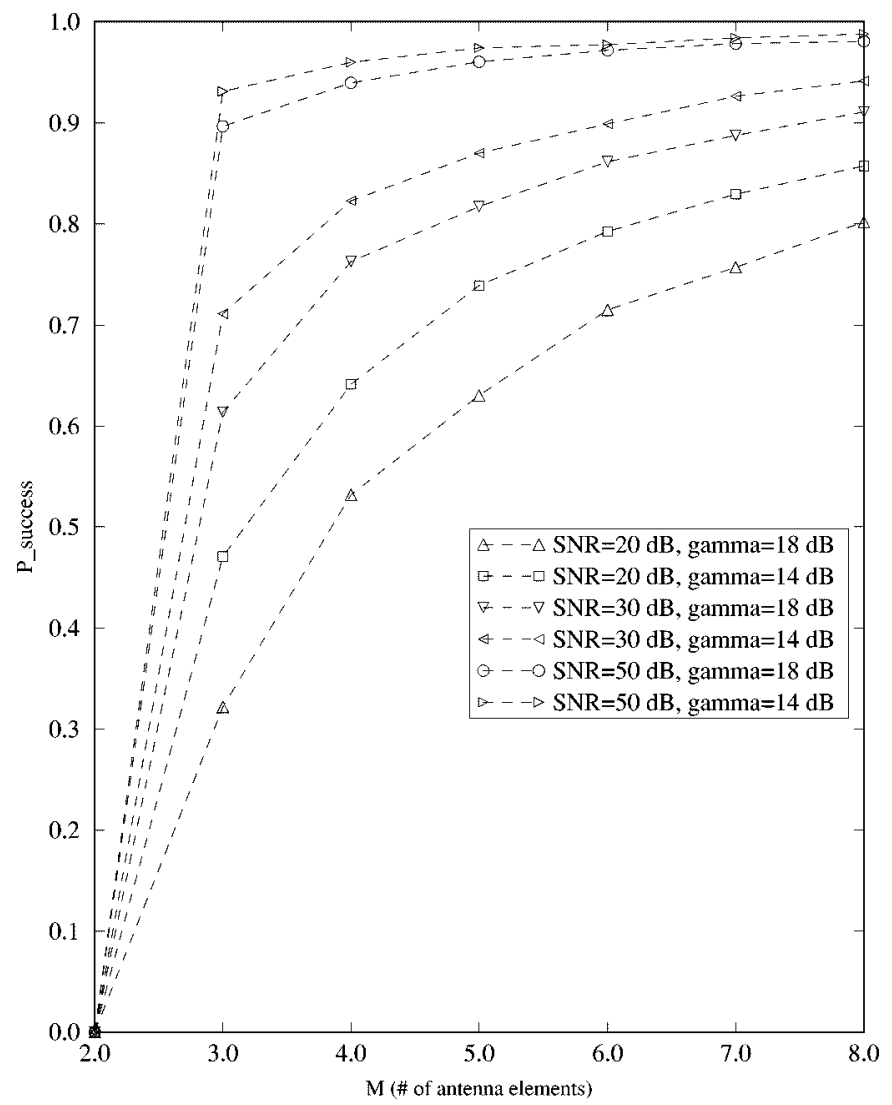

Fig. 8. Success probability $p_{3 \mid 2, M}$ for a three-beam adaptive array. 
for $k=2, \ldots, K$. Furthermore, based on our channel assignment policy illustrated in Fig. $6, x_{K} \leq \cdots \leq x_{3} \leq x_{2}$. Essentially, the channel occupancy vector represents the number of calls assigned to beamformed channels. From Fig. 6, it is easy to show that first $L$ incoming calls will be assigned to the $L$ physical channels with success probability $q_{n+1 \mid n}=1$ for $n=0,1, \ldots, L-1$. If there are more than $L$ simultaneous calls in a cell, they are served by beamformed channels.

As an example, consider a three-beam adaptive array with $L+2$ users in the system. Then the channel occupancy vector $\left(x_{2}, x_{3}\right)$ could be either $(2,0)$ or $(1,1)$. Fig. 9(a) and (b) illustrates this situation. Now there are two possibilities for the transition from $L+2$ to $L+3$ users in the system, i.e., new channel occupancy vector $\left(\hat{x}_{2}, \hat{x}_{3}\right)$ could be either $(3,0)$ or $(2$, 1). Fig. 9(c) and (d) illustrates this situation.

Now for the general case, let $n(n>L)$ denote the number of users in the system at time $t$, i.e., $x_{2}+x_{3}+\cdots+x_{K}=$ $n-L$. Then the number of users in the system at time $t+1$ after the transition (accepting one new call into the system) satisfies $\hat{x}_{2}+\hat{x}_{3}+\cdots+\hat{x}_{K}=n+1-L$, where $\left(\hat{x}_{2}, \hat{x}_{3}, \ldots, \hat{x}_{K}\right)$ denotes new channel occupancy vector, and $\hat{x}_{K} \leq \cdots \leq \hat{x}_{3} \leq \hat{x}_{2}$. The channel assignment policy illustrated in Fig.6 starts from the channel with smallest index $(i=0,1, \ldots, L)$, which has the smallest number of beams $(k=0,1, \ldots, K)$. The first beamformed channel that satisfies the link quality will be assigned to the new call.

Let $\hat{q}\left(n+1, x 2, \ldots, x_{K}\right)$ denote the probability of successful admission of the $(n+1)$ th call into the system, i.e., $\hat{x}_{2}+\hat{x}_{3}+$ $\cdots+\hat{x}_{K}=n+1-L$ with $\hat{x}_{K} \leq \cdots \leq \hat{x}_{3} \leq \hat{x}_{2}$, given $\left(x_{2}, x_{3}, \ldots, x_{K}\right)$ where $x_{2}+x_{3}+\cdots+x_{K}=n-L$ and $x_{K} \leq \cdots \leq x_{3} \leq x_{2}$.

From (20), shown at the bottom of the page, we can compute the success probabilities $q_{n+1 \mid n}$ for the $K$-beam adaptive array as follows:

$$
\begin{aligned}
q_{n+1 \mid n}= & \sum_{\left\{x_{2} \cdots x_{K} \mid x_{K} \leq \cdots \leq x_{2}\right\}} \hat{q}\left(n+1, x_{2}, \ldots, x_{K}\right) \\
& \times p\left[\left(x_{2}, \ldots, x_{K}\right) \mid x_{2}+\cdots+x_{K}=n-L\right] \\
= & \sum_{\substack{\left\{x_{2} \cdots x_{K} \mid x_{K} \leq \cdots \leq x_{2}\right\}\\
}} \hat{q}\left(n+1, x_{2}, \ldots, x_{K}\right) \\
& \times \frac{p\left[\left(x_{2}, \ldots, x_{K}\right), x_{2}+\cdots+x_{K}=n-L\right]}{p\left[x_{2}+\cdots+x_{K}=n-L\right]} .
\end{aligned}
$$

In view of (21), success probabilities $q_{n+1 \mid n}$ can be computed recursively starting from state $\left(x_{2}, x_{3}, \ldots, x_{K}\right)=$ $(1,0, \ldots, 0)$. These results will be used in the numerical results section to compare traffic/throughput improvements in a system with two- and three-beam adaptive arrays with that of a system with a single omnidirectional antenna.

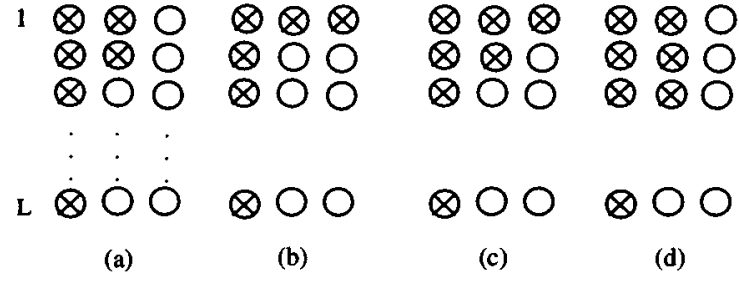

Fig. 9. Channel occupancy scenarios for a three-beam array, when there are $L+2$ users in the system (a) Two users are served by channels in the second beam. (b) One user with the second beam and another one with the third beam are being served. Transition of system to $L+3$ users. (c) Two users with the second beam and one with the third beam are being served. (d) Three users are served with the second beam.

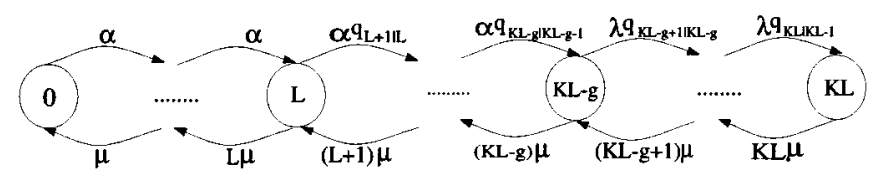

Fig. 10. State transition diagram of discrete Markov chain, the network model of the system with an adaptive array at the base station and guard channels.

In our network model setup, the effective arrival rate into the cell at state $n$ is [19]

$$
\lambda_{\text {eff }}(n)=\left\{\begin{array}{ll}
\alpha q_{n+1 \mid n} & n=0,1, \ldots, K L-g-1 \\
\lambda q_{n+1 \mid n} & n=K L-g, \ldots, K L-1 .
\end{array} .\right.
$$

Similarly, the effective service rate in the system at a given state $n, \mu(n)$, is

$$
\mu(n)=n \mu \quad n=1,2, \ldots, K L .
$$

Let $n, n=0,1, \ldots K L$, denote the number of users served in a wireless network using adaptive antenna arrays. The number of users $n$ in such a system forms a Markov chain with state transition diagram shown in Fig. 10.

It is worth mentioning that the network model in [5], which assumes an omnidirectional antenna at the base station will be a special case of our generalized model with $K=1$, with $q_{n+1 \mid n}=1$ for $n=0,1, \ldots, L-1$, which results in the same model presented in Section III. Using the results of queuing theory [19], [20] we have

$$
P(n+1)=\frac{\lambda_{\mathrm{eff}}(n)}{\mu(n+1)} P(n) \text { for } n=0,1, \ldots, K L-1 .
$$

From (24), it readily follows that

$$
P(n)=\prod_{j=0}^{n-1} \frac{\lambda_{\mathrm{eff}}(j)}{(j+1) \mu} P(0) \quad \text { for } n=0,1, \ldots, K L .
$$

$$
\begin{aligned}
\hat{q}\left(n+1, x_{2}, \ldots, x_{K}\right)= & p\left[\hat{x}_{2}+\cdots+\hat{x}_{K}=n+1-L \mid\left(x_{2}, \ldots, x_{K}\right), x_{2}+\cdots+x_{K}=n-L\right] \\
= & 1-\left(1-p_{2 \mid 1, M}\right)^{\left(L-x_{2}\right)} \times\left(1-p_{3 \mid 2, M}\right)^{\left(x_{2}-x_{3}\right)} \\
& \times \cdots \times\left(1-p_{K \mid K-1, M}\right)^{\left(x_{K-1}-x_{K}\right)}
\end{aligned}
$$


Now from (22), (23), and (25), the state probabilities $P(n)$ are shown by

$$
\begin{aligned}
& P(n)= \\
& \begin{cases}\frac{a^{n}}{n !} \prod_{j=0}^{n-1} q_{j+1 \mid j} P(0) & n=0,1, \ldots, K L-g \\
\frac{a^{K L-g} c^{n-(K L-g)}}{n !} \prod_{j=0}^{n-1} q_{j+1 \mid j} P(0) & n=K L-g, \ldots, K L .\end{cases}
\end{aligned}
$$

where $P(0)$ is

$$
\begin{aligned}
P(0)= & {\left[\sum_{n=0}^{K L-g-1} \frac{a^{n}}{n !} \prod_{j=0}^{n-1} q_{j+1 \mid j}+a^{K L-g}\right.} \\
& \left.\cdot \sum_{n=K L-g}^{K L} \frac{c^{n-(K L-g)}}{n !} \prod_{j=0}^{n-1} q_{j+1 \mid j}\right]^{-1} .
\end{aligned}
$$

The blocking probability $B_{O}$ of originating calls is then given by

$$
\begin{aligned}
B_{O}= & \sum_{n=L}^{K L-g-1}\left(1-q_{n+1 \mid n}\right) P(n)+P[n \geq K L-g] \\
\Rightarrow B_{O}= & {\left[\sum_{n=L}^{K L-g-1}\left(1-q_{n+1 \mid n}\right) \frac{a^{n}}{n !} \prod_{j=0}^{n-1} q_{j+1 \mid j}\right.} \\
& \left.+a^{K L-g} \sum_{n=K L-g}^{K L} \frac{c^{n-(K L-g)}}{n !} \prod_{j=0}^{n-1} q_{j+1 \mid j}\right] P(0) .
\end{aligned}
$$

As we can see from (28), blocking probability of originating calls consists of two terms. The term $P[n \geq K L-g]$ is due to the fact that originating calls are blocked if there are $g$ or less free channels in the system. The term $\sum_{n=L}^{K L-g-1}\left(1-q_{n+1 \mid n}\right) P(n)$ in (28) represents the blocking probabiltiy due to unsuccessful beamformings.

Similarly, for the handoff calls, we have the blocking probability $B_{H}$ given by

$$
B_{H}=\sum_{n=L}^{K L-1}\left(1-q_{n+1 \mid n}\right) P(n)+P[n=K L]
$$

$$
\begin{aligned}
\Rightarrow B_{H}= & {\left[\sum_{n=L}^{K L-g-1}\left(1-q_{n+1 \mid n}\right) \frac{a^{n}}{n !} \prod_{j=0}^{n-1} q_{j+1 \mid j}\right.} \\
& +\sum_{n=K L-g}^{K L-1}\left(1-q_{n+1 \mid n}\right) \frac{a^{K L-g} c^{n-(K L-g)}}{n !} \prod_{j=0}^{n-1} q_{j+1 \mid j} \\
& \left.+\frac{a^{K L-g} C^{g}}{(K L) !} \prod_{j=0}^{K L-1} q_{j+1 \mid j}\right] P(0) .
\end{aligned}
$$

As mentioned, in a network with an omnidirectional antenna, a handoff call is blocked if and only if all channels in the cell happen to be busy. In a wireless network with smart antennas at the base station, there is a possibility of blocking handoff calls due to unsuccessful beamformings. This effect is taken into account in (29) through $\sum_{n=L}^{K L-1}\left(1-q_{n+1 \mid n}\right) P(n)$. The second term in (29), $P[n=K L]$, is the contribution to the blocking probability when all the beamformed channels are busy. In the numerical results section, the blocking probabilities given by (17) and (18) will be compared to those derived for the network with an adaptive array at the base station given by (28) and (29).

\section{Network with Adaptive ArRay and Queuing of HANDOFF CALLS}

In this section, we investigate other traffic policies that further decrease the blocking probability of handoff calls at the expense of slightly increasing the blocking probability of originating calls [5], [21]. We first assume that infinite queues are allowed for handoff calls. The state balance equations can then be easily derived. Three distinct cases have to be distinguished, corresponding to the three possible behavior of the system.

1) All customers are served without any distinction of type.

2) Originating calls are blocked and handoff calls are served.

3) Originating calls are blocked and handoff calls are queued.

More precisely, we have the following relations:

$$
\left\{\begin{array}{ll}
1.0 \leq n \leq K L-g-1 & P(n+1)=\frac{a q_{n+1 \mid n}}{n+1} P(n) \\
\text { 2. } K L-g \leq n \leq K L-1 & P(n+1)=\frac{c q_{n+1 \mid n}}{n+1} P(n) \\
\text { 3. } n \geq K L & P(n+1)=\frac{c+1}{K L} P(n)
\end{array} .\right.
$$

From the above relations between the state probabilities obtained from the state transitions, we easily derive the exact ex-

$$
\begin{gathered}
\begin{cases}0 \leq n \leq K L-g, & P(n)=\frac{a^{n}}{n !} \prod_{j=0}^{n-1} q_{j+1 \mid j} P(0) \\
K L-g \leq n \leq K L, & P(n)=\frac{a^{K L-g} c^{n-(K L-g)}}{n !} \prod_{j=0}^{n-1} q_{j+1 \mid j} P(0) \\
n \geq K L, & P(n)=\frac{a^{K L-g} c^{n-(K L-g)}}{(K L) !(K L)^{n-K L}} \prod_{j=0}^{K L-1} q_{j+1 \mid j} P(0)\end{cases} \\
\frac{1}{P(0)}=\sum_{n=0}^{K L-g-1} \frac{a^{n}}{n !} \prod_{j=0}^{n-1} q_{j+1 \mid j}+a^{K L-g} \sum_{n=K L-g}^{K L-1} \frac{c^{n-(K L-g)}}{n !} \prod_{j=0}^{n-1} q_{j+1 \mid j}+\frac{a^{K L-g} C^{g}}{(K L-1) !(K L-c)} \prod_{j=0}^{K L-1} q_{j+1 \mid j}
\end{gathered}
$$


pressions of the state probabilities. Namely, we get (31), shown at the bottom of the page, where $P(0)$ is given by (32) at the bottom of the page. Being now provided with the state probabilities as given by (31), we can compute the blocking probability for originating calls, as shown in (33) at the bottom of the page. We can remark that due to the fact that handoff calls are allowed to be queued, $B_{O}^{\prime}$ is slightly greater than the previous $B_{O}$, even in the case $g=0$, because all handoff calls present in the queue will be served prior to any originating call. This will be more illustrated in the numerical results section. Furthermore, the fact that we assume infinite queuing capacity for handoff calls implies that handoff calls will be blocked only due to unsuccessful capturing after beamforming process. This blocking probability would beIn the case that the number of active calls in the cell exceeds $K L$, any new handoff call will be queued in an infinite queue. We will treat the more realistic case of finite queue length later, and we will also estimate the average waiting time experienced by handoff calls. Since handoff calls are queued, we shall compute the probability that a handoff call being delayed. This quantity is easily obtained from the state probabilities given in (31) by (35), shown at the bottom of the page.

We can also obtain the probability of a handoff's being delayed more than a certain time $t$. This probability is the sum over all possible values $n \geq K L$ of finding $n$ customers in the system, times the probability that there are fewer than $n-K L$ departures from the system in time $t$. The departure process from the system when all servers are busy is a Poisson process with rate $K L \mu$, i.e., see (36) at the bottom of the page.

We start the calculation by computing the density function of the waiting time $w(t)$ of a handoff call. Namely, a handoff call will have a waiting time between $t$ and $t+d t$ if it finds $n \geq K L$ customers in the system upon its arrival, if $(n-K L)$ depart within time $t$, and if another one departs between time $t$ to $t+d t$. For a given value of $n \geq K L$, this probability is given by

$$
\begin{aligned}
\operatorname{Pr}\{w \in[t, t+d t] \mid n & \geq K L\} \\
& =K L \mu \frac{(K L \mu t)^{n-K L}}{(n-K L) !} e^{-K L \mu t} d t .
\end{aligned}
$$

The expression for the density function $w(t)$ of the waiting time of a handoff call is obtained by summing for all values of $n$ in the above expression multiplied by the probability of finding $n \geq K L$ customers in the system, as shown in (38) at the bottom of the next page. After integrating (38), we obtain the probability that a handoff call waits more than a certain time $t$. Namely, we get

$$
\begin{aligned}
P_{H}(>t) & =\int_{t}^{\infty} w(t) d t \\
& =\frac{a^{K L-g} c^{g}}{(K L-1) !(K L-c)} e^{-\mu t(K L-c)} \prod_{j=0}^{K L-1} q_{j+1 \mid j} P(0) \\
& =e^{-\mu t(K L-c)} \times P_{H}(>0)
\end{aligned}
$$

where $P_{H}(>0)$ is given by (35). It is interesting to note that the waiting time turns out to be conditionally memoryless, given that the system is busy. From (38), the average delay for handoff calls is

$$
\begin{aligned}
W_{H} & =\int_{0}^{\infty} t w(t) d t \\
& =\frac{a^{K L-g} c^{g}}{(K L-1) !(K L-c)^{2} \mu} \prod_{j=0}^{K L-1} q_{j+1 \mid j} P(0) \\
& =\frac{1}{\mu(K L-c)} \times P_{H}(>0) .
\end{aligned}
$$

We recall that we decided to introduce the possibility of queuing handoff calls, in order to further decrease their blocking proba-

$$
\begin{aligned}
& B_{O}^{\prime}=\sum_{n=L}^{K L-g-1}\left(1-q_{n+1 \mid n}\right) P(n)+P(n \geq K L-g) \\
& \Rightarrow B_{O}^{\prime}=\left[\sum_{n=L}^{K L-g-1}\left(1-q_{n+1 \mid n}\right) \frac{a^{n}}{n !} \prod_{j=0}^{n-1} q_{j+1 \mid j}+\left(\frac{a}{c}\right)^{K L-g} \sum_{n=K L-g}^{K L-1} \frac{c^{n}}{n !} \prod_{j=0}^{n-1} q_{j+1 \mid j}+\frac{a^{K L-g} c^{g}}{(K L-1) !(K L-c)} \prod_{j=0}^{K L-1} q_{j+1 \mid j} P(0)\right. \\
& \begin{aligned}
B_{H}^{\prime} & =\sum_{n=L}^{K L-1}\left(1-q_{n+1 \mid n}\right) P(n) \\
\Rightarrow B_{H}^{\prime} & =\left[\sum_{n=L}^{K L-g-1}\left(1-q_{n+1 \mid n}\right) \frac{a^{n}}{n !} \prod_{j=0}^{n-1} q_{j+1 \mid j}+\sum_{n=K L-g}^{K L-1}\left(1-q_{n+1 \mid n}\right) \frac{a^{K L-g} c^{n-(K L-g)}}{n !} \prod_{j=0}^{n-1} q_{j+1 \mid j}\right] P(0)
\end{aligned} \\
& P\{\text { handoff call is delayed }\}=P\{n \geq K L\}=P_{H}(>0) \\
& \Rightarrow P_{H}(>0)=\frac{a^{K L-g_{C} g}}{(K L-1) !(K L-c)} \prod_{j=0}^{K L-1} q_{j+1 \mid j} P(0) \text {. }
\end{aligned}
$$

$\operatorname{Pr}\{(n-K L)$ departures in the system in $t$ seconds $\mid n \geq K L\}=\frac{(K L \mu t)^{n-K L}}{(n-K L) !} e^{-K L \mu t}$ 
bility, but we were also hoping for a small average waiting time. An example of the behavior of the average waiting time of the handoff calls in a network with adaptive array at the base station is provided in the numerical results section. From these results, we can see that guard channels are efficient in decreasing the average waiting time of handoff calls, making the possibility of queuing handoff calls useful.

However, what we are really interested in is the average delay of handoff calls that actually do experience a delay. This conditional delay is simply obtained by dividing the overall average delay $W_{H}$ given by (40) by the probability of being delayed $P_{H}(>0)$ given by (35). This quantity turns out to be independent of the number of the guard channels $g$ and is equal to

$$
D_{H}=\frac{1}{\mu(K L-c)} .
$$

Now it is clear that increasing the number of guard channels will only decrease the number of handoff calls that have to be queued, without reducing their average delay. An important result implied by (41) is that $D_{H}$ is inversely proportional to the number of adaptive beams $K$. For instance, if $K L \gg c$, $D_{H} \approx 1 / \mu K L$. Therefore, the average delay experienced by handoff calls in a network with $K$-beam adaptive array is $K$ times smaller than that of the network with a single omnidirectional antenna. The need for a small waiting time comes from the fact that we want the queuing process to go nearly unnoticed by a customer involved in a call.

We now investigate the case where queuing is again allowed, but the queue size is more realistically taken to be finite, equal to some integer $Q$. We are hoping that the queue size limitation might help decrease the average waiting time of calls that are delayed, since long delays come from customers that find a large queue ahead of them upon their arrival. However, limiting the size implies that a blocking probability for handoff calls will again appear. The only change in the state probabilities is that they are now equal to zero for $n$ strictly greater than $K L+Q$. On the other hand, the state probabilities for $0 \leq n \leq K L+Q$ are still given by (31), and the only modification needed is the expression for $P(0)$. Namely, we have (42), shown at the bottom of the page, where $P_{Q}(0)$ is given by

$$
\begin{aligned}
\frac{1}{P_{Q}(0)}= & {\left[\sum_{n=0}^{K L-g-1} \frac{a^{n}}{n !} \prod_{j=0}^{n-1} q_{j+1 \mid j}+\left(\frac{a}{c}\right)^{K L-g}\right.} \\
& \cdot \sum_{n=K L-g}^{K L-1} \frac{c^{n}}{n !} \prod_{j=0}^{n-1} q_{j+1 \mid j} \\
& \left.+\frac{a^{K L-g} c^{g}}{(K L-1) !} \prod_{j=0}^{K L-1} q_{j+1 \mid j} \times \frac{1-\left(\frac{c}{K L}\right)^{Q+1}}{K L-c}\right] .
\end{aligned}
$$

$$
\begin{aligned}
w(t) d t & =\sum_{n=K L}^{\infty} P(n) \operatorname{Pr}\{w \in[t, t+d t] \mid n \geq K L\} \\
& =\frac{a^{K L-g} c^{g}}{(K L) !} e^{-K L \mu t} K L \mu d t P(0) \times \sum_{n=K L}^{\infty}\left(\frac{c}{K L}\right)^{n-K L} \prod_{j=0}^{K L-1} q_{j+1 \mid j} \times \frac{(K L \mu t)^{n-K L}}{(n-K L) !} \\
& =\frac{a^{K L-g} C^{g}}{(K L) !} e^{-K L \mu t} K L \mu d t \prod_{j=0}^{K L-1} q_{j+1 \mid j} P(0) \sum_{n=K L}^{\infty} \frac{(c \mu t)^{n-K L}}{(n-K L) !} \\
& =\frac{a^{K L-g} c^{g}}{(K L) !} e^{-K L \mu t} K L \mu d t \prod_{j=0}^{K L-1} q_{j+1 \mid j} P(0) \sum_{i=0}^{\infty} \frac{(c \mu t)^{i}}{i !} \\
& =\frac{a^{K L-g} C^{g}}{(K L) !} e^{-K L \mu t} K L \mu d t \prod_{j=0}^{K L-1} q_{j+1 \mid j} P(0) e^{c \mu t} \\
\Rightarrow w(t) & =\frac{a^{K L-g} C^{g}}{(K L-1) !} e^{-\mu t(K L-c)} \mu \prod_{j=0}^{K L-1} q_{j+1 \mid j} P(0)
\end{aligned}
$$

$$
\begin{cases}0 \leq n \leq K L-g & P(n)=\frac{a^{n}}{n !} \prod_{j=0}^{n-1} q_{j+1 \mid j} P_{Q}(0) \\ K L-g \leq n \leq K L & P(n)=\frac{a^{K L-g} c^{n-(K L-g)}}{n !} \prod_{j=0}^{n-1} q_{j+1 \mid j} P_{Q}(0) \\ K L \leq n \leq K L+Q & P(n)=\frac{a^{K L-g} c^{n-(K L-g)}}{(K L) !(K L)^{n-K L}} \prod_{j=0}^{K L-1} q_{j+1 \mid j} P_{Q}(0) \\ n>K L+Q & P(n)=0\end{cases}
$$


Now the blocking probability for originating calls $B_{\mathrm{OO}}^{\prime}$ (where $Q$ denotes the length of the queue) is equal to

$$
\begin{aligned}
B_{\mathrm{QO}}^{\prime}= & \sum_{n=L}^{K L-g-1}\left(1-q_{n+1 \mid n}\right) P(n)+P(n \geq K L-g) \\
\Rightarrow B_{\mathrm{QO}}^{\prime}= & {\left[\sum_{n=L}^{K L-g-1}\left(1-q_{n+1 \mid n}\right) \frac{a^{n}}{n !} \prod_{j=0}^{n-1} q_{j+1 \mid j}+\left(\frac{a}{c}\right)^{K L-g}\right.} \\
& \sum_{n=K L-g}^{K L-1} \frac{c^{n}}{n !} \prod_{j=0}^{n-1} q_{j+1 \mid j} \\
& \left.+\frac{a^{K L-g_{c} g}}{(K L-1) !} \prod_{j=0}^{K L-1} q_{j+1 \mid j} \times \frac{1-\left(\frac{c}{K L}\right)^{Q+1}}{K L-c}\right] P_{Q}(0) .
\end{aligned}
$$

As a check, (44) reduces to (33) if we let $Q \rightarrow \infty$ and to (28) if $Q=0$. Similarly, for handoff calls we can compute the blocking probability $B_{\mathrm{QH}}^{\prime}$ introduced by the finite queue length $Q$. We have

$$
\begin{aligned}
B_{\mathrm{QH}}^{\prime}= & \sum_{n=L}^{K L-1}\left(1-q_{n+1 \mid n}\right) P(n)+P[n=K L+Q] \\
\Rightarrow B_{\mathrm{QH}}^{\prime}= & {\left[\sum_{n=L}^{K L-g-1}\left(1-q_{n+1 \mid n}\right) \frac{a^{n}}{n !} \prod_{j=0}^{n-1} q_{j+1 \mid j}\right.} \\
& +\sum_{n=K L-g}^{K L-1}\left(1-q_{n+1 \mid n}\right) \frac{a^{K L-g} c^{n-(K L-g)}}{n !} \\
& \cdot \prod_{j=0}^{n-1} q_{j+1 \mid j}+\frac{a^{K L-g} c^{Q+g}}{(K L) !(K L)^{Q}} \\
& \left.\cdot \prod_{j=0}^{K L-1} q_{j+1 \mid j}\right] P_{Q}(0) .
\end{aligned}
$$

Again, we can check that (45) reduces to (34) if $Q \rightarrow \infty$ and reduces to (29) if we let $Q=0$. We can also derive the probability for a handoff call to be delayed, as shown in (46) at the bottom of the page. Equation (46) gives zero for $Q=0$ and reduces to (35) if we let $Q \rightarrow \infty$ as we expected. Last, we want the probability that a handoff call is delayed more that $t$ seconds. As in the case of infinite queue length, we start by deriving the density function of the waiting time of handoff calls. The computations are completely parallel to the one that led to (38) except for the fact that we now only consider the case $K L \leq n<K L+Q$; we get

$$
\begin{aligned}
w_{Q}(t)= & P_{Q}(0) \frac{a^{K L-g_{C} g}}{(K L) !} K L \mu \\
& \cdot \prod_{j=0}^{K L-1} q_{j+1 \mid j} e^{-K L \mu t} \sum_{i=0}^{Q-1} \frac{(c \mu t)^{i}}{i !} .
\end{aligned}
$$

After integration, this gives the desired expression for the probability that a handoff call be delayed more than $t$

$$
\begin{aligned}
P_{\mathrm{QH}}(>t) & =P_{Q}(0) \frac{a^{K L-g} c^{g}}{(K L) !} \\
& \times \prod_{j=0}^{K L-1} q_{j+1 \mid j} \sum_{i=0}^{Q-1} \int_{t}^{\infty} K L \mu e^{-K L \mu x} \frac{(c \mu x)^{i}}{i !} d x \\
P_{\mathrm{QH}}(>t) & =P_{Q}(0) \frac{a^{K L-g} c^{g}}{(K L) !} \\
& \times \prod_{j=0}^{K L-1} q_{j+1 \mid j} e^{-K L \mu t} \sum_{i=0}^{Q-1}\left(\sum_{j=0}^{i} \frac{(c \mu t)^{i-j}}{(i-j) !}\left(\frac{c}{K L}\right)^{j}\right) .
\end{aligned}
$$

Equation (48) can be checked to reduce to (38) when we let $Q \rightarrow \infty$. This is best seen by realizing that for $L \rightarrow \infty$ the expression $w_{Q}(t)$ gives the same expression as (38), since we have

$$
\lim _{Q \rightarrow \infty}\left(\sum_{i=0}^{Q-1} \frac{(c \mu t)^{i}}{i !}\right)=e^{c \mu t} .
$$

From (47), we can also deduce the expression of the average waiting time $W_{\mathrm{QH}}$ of handoff calls. Namely, we have

$$
\begin{aligned}
W_{\mathrm{QH}}= & \int_{0}^{\infty} t w_{Q}(t) d t \\
= & P_{Q}(0) \frac{a^{K L-g} c^{g}}{(K L) !} \prod_{j=0}^{K L-1} q_{j+1 \mid j} \\
& \cdot \sum_{i=0}^{Q-1} \int_{0}^{\infty} K L \mu t e^{-K L \mu t} \frac{(c \mu t)^{i}}{i !} d t \\
= & P_{Q}(0) \frac{a^{K L-g} c^{g}}{(K L) !} \frac{1}{c \mu} \prod_{j=0}^{K L-1} q_{j+1 \mid j} \\
& \cdot \sum_{i=0}(i+1)\left(\frac{c}{K L}\right)^{i+1} .
\end{aligned}
$$

$$
\begin{aligned}
P\{\text { handoff call is delayed }\} & =P[K L \leq n<K L+Q]=P_{\mathrm{QH}}(>0) \\
\Rightarrow P_{\mathrm{QH}}(>0) & =\frac{a^{K L-g} c^{g}}{(K L-1) !} \times \prod_{j=0}^{K L-1} q_{j+1 \mid j} \frac{1-\left(\frac{c}{K L}\right)^{Q}}{K L-c} P_{Q}(0)
\end{aligned}
$$




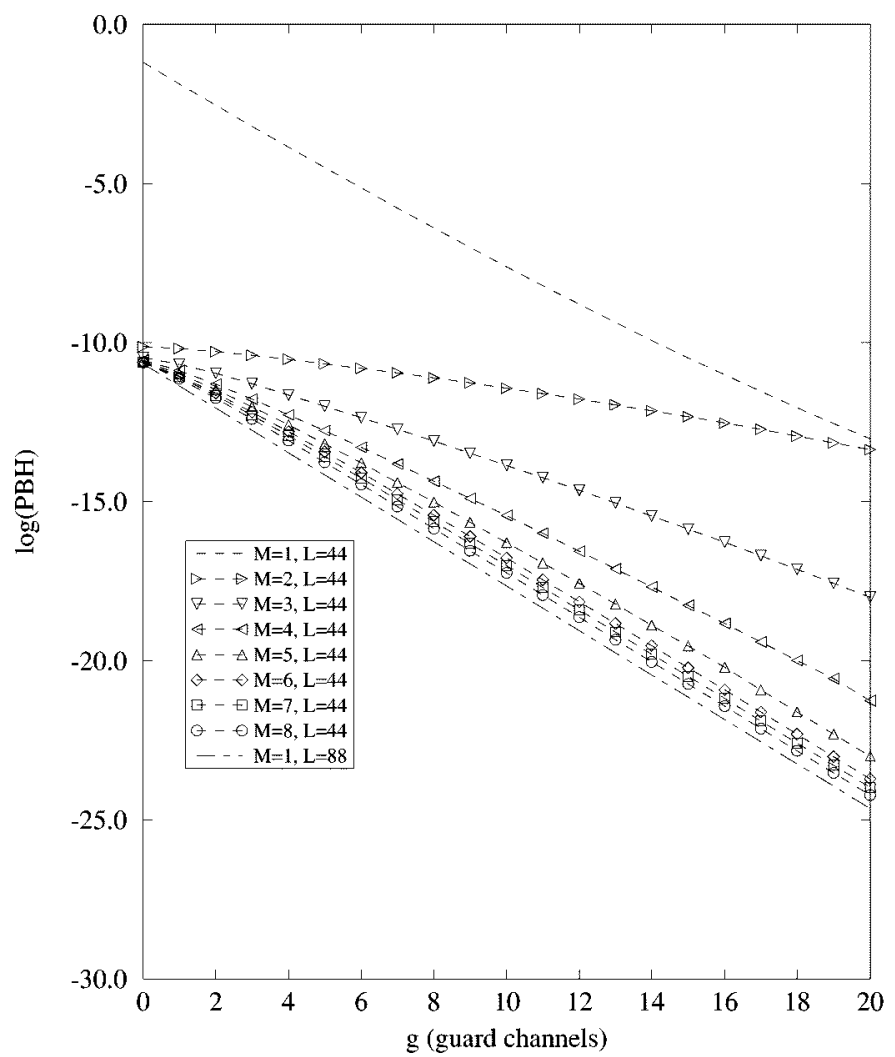

(a)

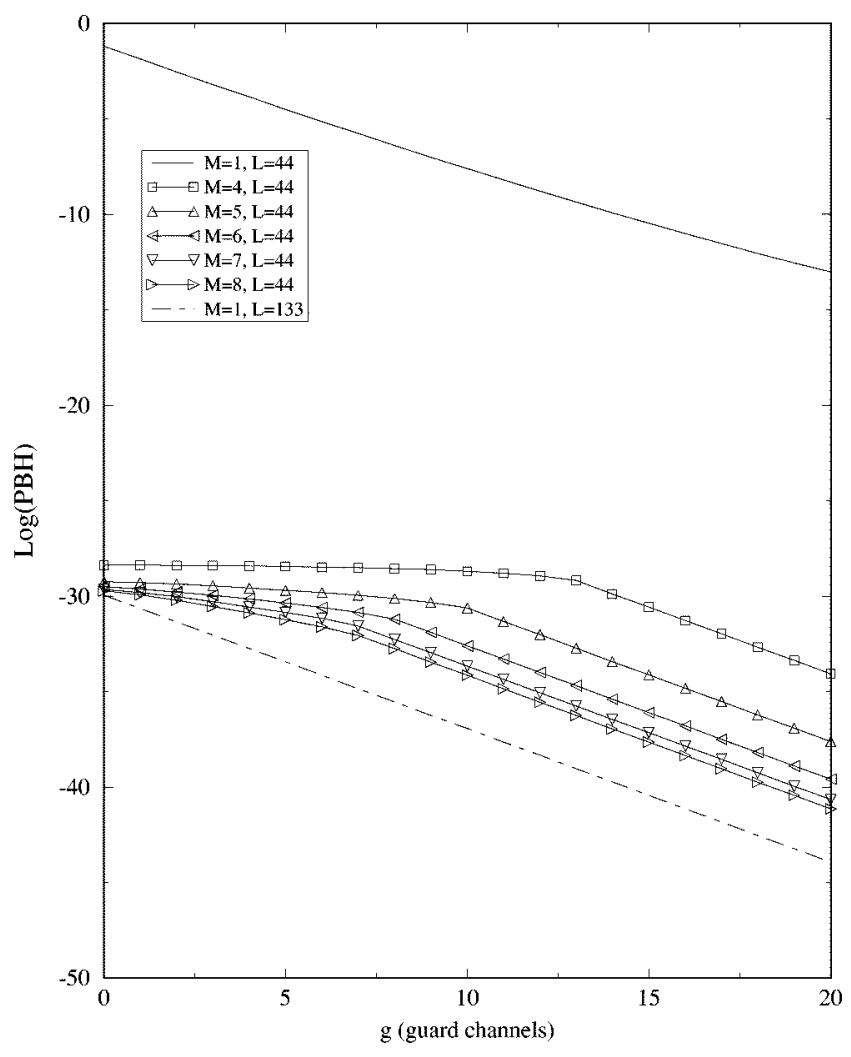

(b)

Fig. 11. Blocking probabilities of handoff calls $\left(B_{H}\right)$ for different number of antenna elements $M$ with SNR $=20, \gamma=18 \mathrm{~dB}, a=40, c=8, \beta=4$ in a two-beam (top) three-beam adaptive array system.

We can simplify

$$
\begin{aligned}
& \sum_{i=0}^{Q-1}(i+1)\left(\frac{c}{(K L)}\right)^{i+1} \\
& =\frac{c}{K L} \sum_{i=1}^{Q} i\left(\frac{c}{K L}\right)^{i-1} \\
& =c K L \frac{1-(Q+1)\left(\frac{c}{K L}\right)^{Q}+Q\left(\frac{c}{K L}\right)^{Q+1}}{(K L-c)^{2}} .
\end{aligned}
$$

So finally, we get

$$
\begin{aligned}
W_{\mathrm{QH}}= & P_{Q}(0) \frac{a^{K L-g} C^{g} \prod_{j=0}^{K L-1} q_{j+1 \mid j}}{(K L-1) !(K L-c)^{2} \mu} \\
& \cdot\left(1-(Q+1)\left(\frac{c}{K L}\right)^{Q}+Q\left(\frac{c}{K L}\right)^{Q+1}\right)
\end{aligned}
$$

We can now obtain the average delay given handoff calls are delayed $D_{\mathrm{QH}}$ simply by dividing the above expression for $W_{\mathrm{QH}}$ by $P_{\mathrm{QH}}(>0)$, as given in (46). We find that it is again independent of the number of guard channels $g$, as we expected, and is equal to

$$
D_{\mathrm{QH}}=\frac{1}{(K L-c) \mu} \times \frac{1-(Q+1)\left(\frac{c}{K L}\right)^{Q}+Q\left(\frac{c}{K L}\right)^{Q+1}}{1-\left(\frac{c}{K L}\right)^{Q}} .
$$

In Section VI, the average delay of handoff calls for a network with adaptive arrays and finite queue size $D_{\mathrm{QH}}$, given by (53), is to be compared with the average delay of handoff calls with infinite queue size $D_{H}$ given by (41).

\section{NUMERICAL RESULTS}

In this section, we present the numerical results to show the effectiveness of the network with adaptive arrays for traffic/throughput improvements in a wireless network. We evaluate the performance of the system with parameters drawn from [5]. We choose a cell with $L=44$ channels with a total offered traffic $a=40$ Erlangs (heavy traffic) and a handoff traffic $c=8$ Erlangs. The link gain is modeled as $G_{1}=1 / d_{i}^{4}$, where $d_{i}$ is the distance between the base station and the $i$ th mobile.

Figs. 11 and 12 illustrate blocking probabilities given by (18) and (29). From Figs. 11 and 12, it is clear that for a given SNR and threshold $\gamma$, as the number of antenna elements $M$ increases, the $B_{H}$ decreases. These numerical results confirm that using a two-beam (three-beam) adaptive array at the base station, doubles (triples) the system capacity for a reasonably large $M$. Blocking probabilities of originating calls in a three-beam adaptive array system with the same parameters and SNR $=20$ is illustrated in Fig. 13.

Fig. 13 also illustrates the blocking probabilities of handoff calls versus number of antenna elements $M$. From Fig. 13, as $M$ increases, $B_{H}$ decreases rapidly. 


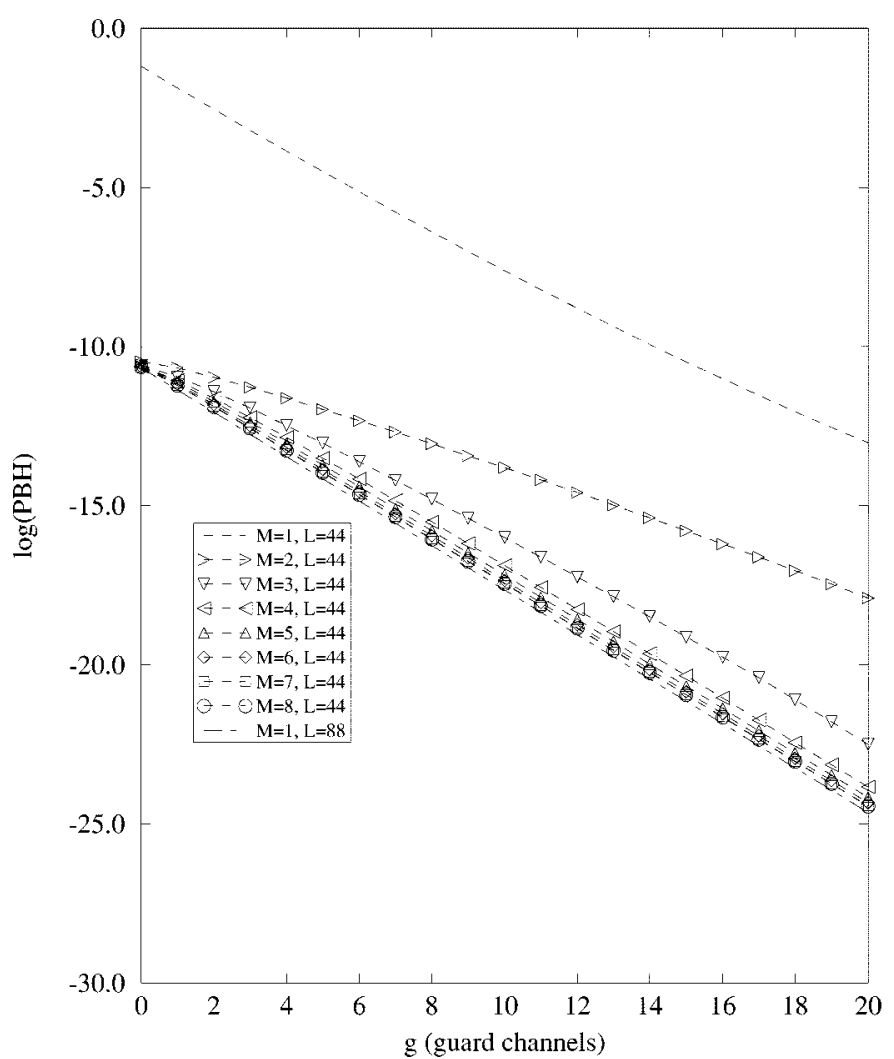

(a)

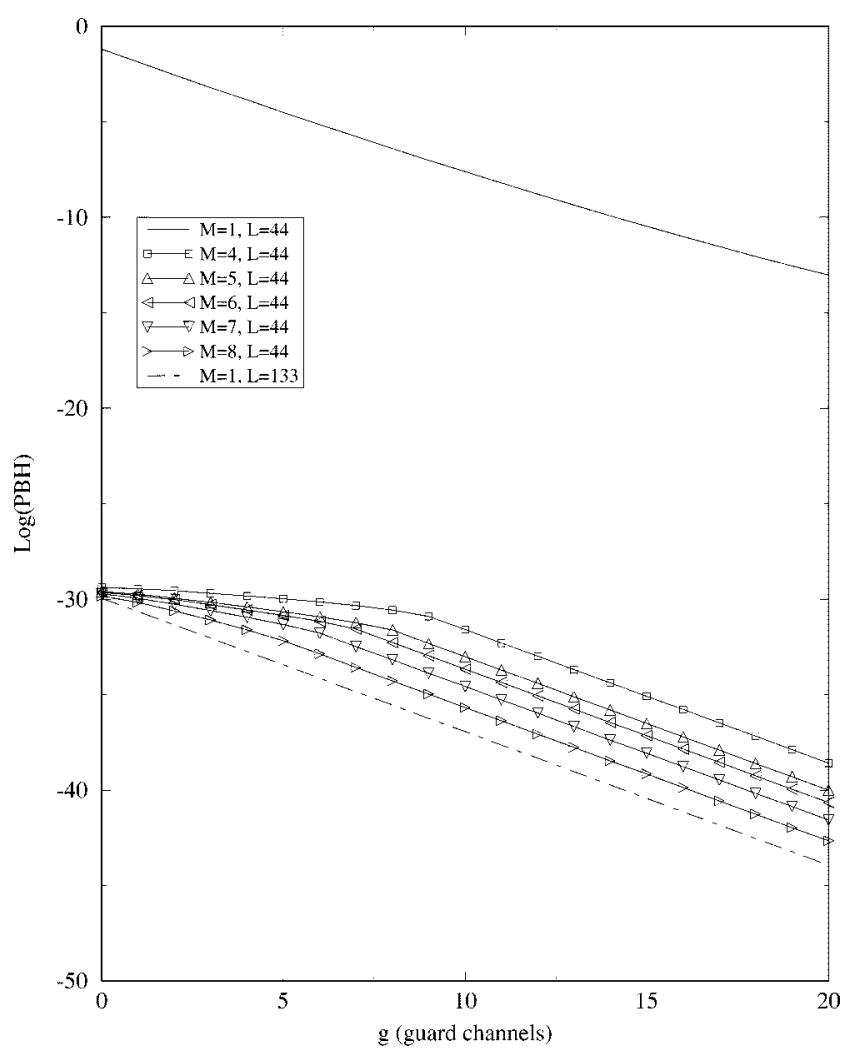

(b)

Fig. 12. Blocking probabilities of handoff calls $\left(B_{H}\right)$ for different number of antenna elements $M$ with SNR $=30, \gamma=18 \mathrm{~dB}, a=40, c=8, \beta=4$ in a two-beam (top) and three-beam adaptive array system.

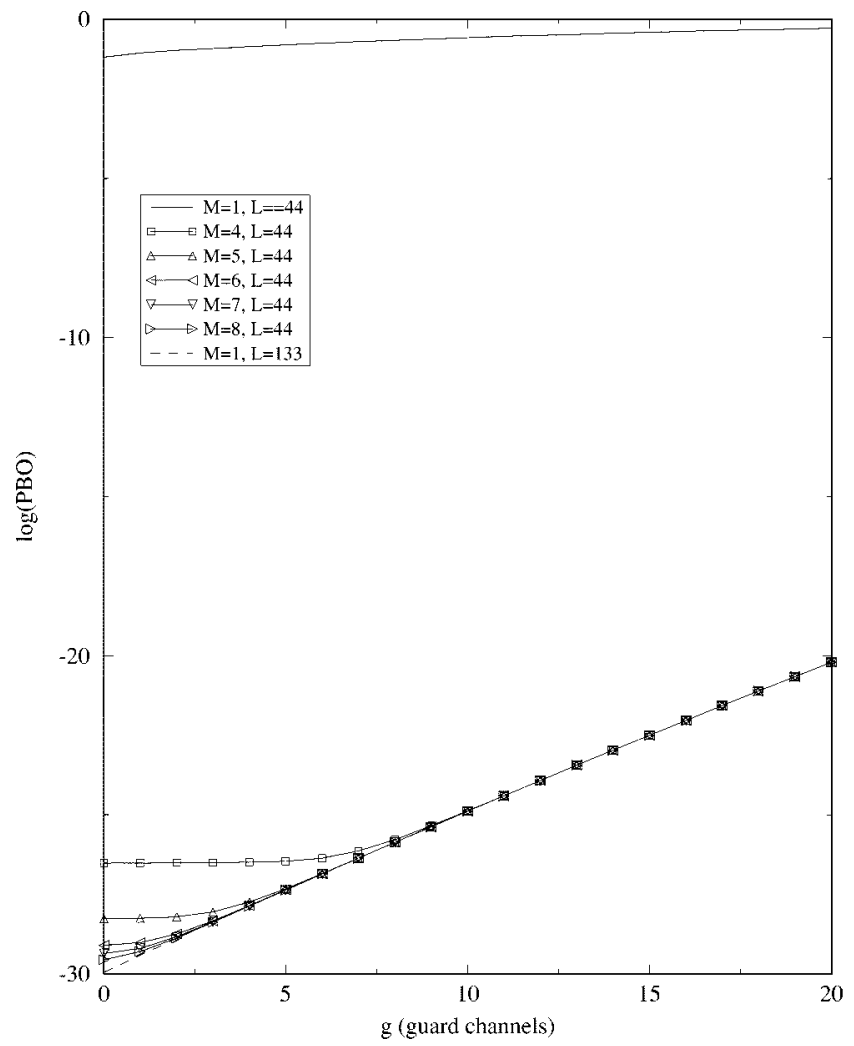

(a)

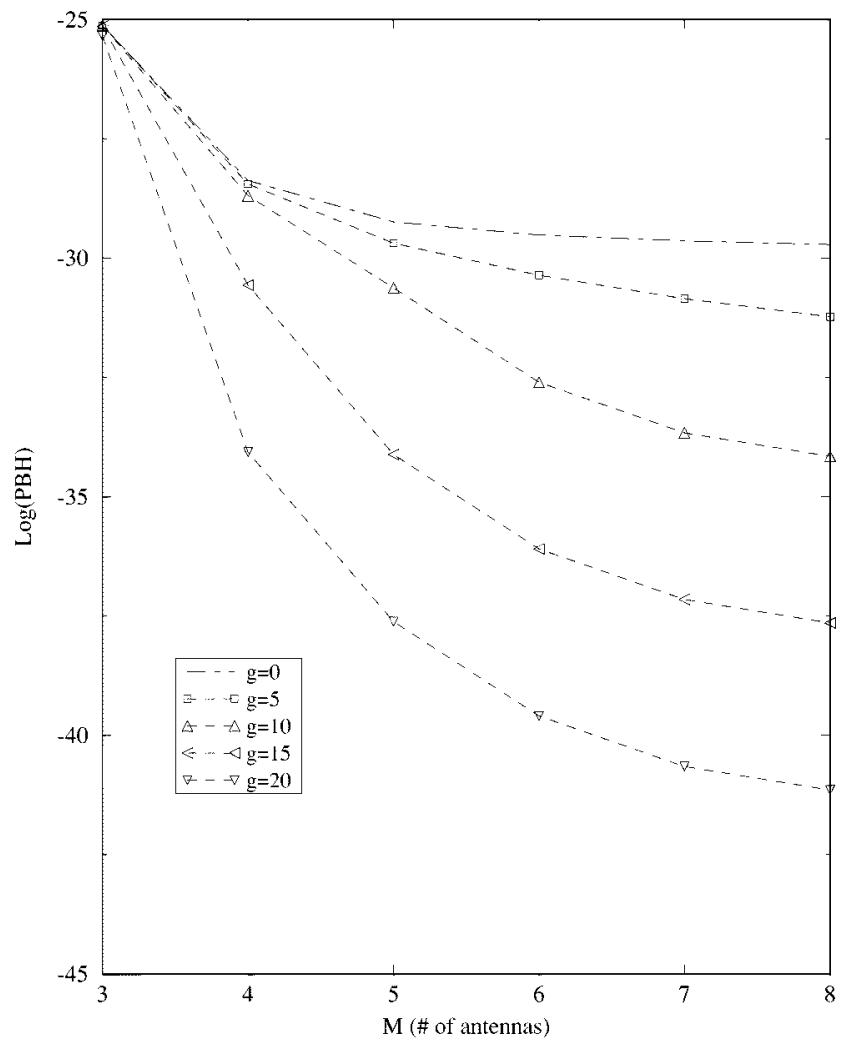

(b)

Fig. 13. Blocking probabilities of originating calls $\left(B_{O}\right)$ for different number of antenna elements $M$ with SNR $=20, \gamma=18 \mathrm{~dB}, a=40, c=8$ in a three-beam adaptive array system (top). Blocking probabilities $B_{H}$ versus number of antenna elements $M$, with the same parameters. 


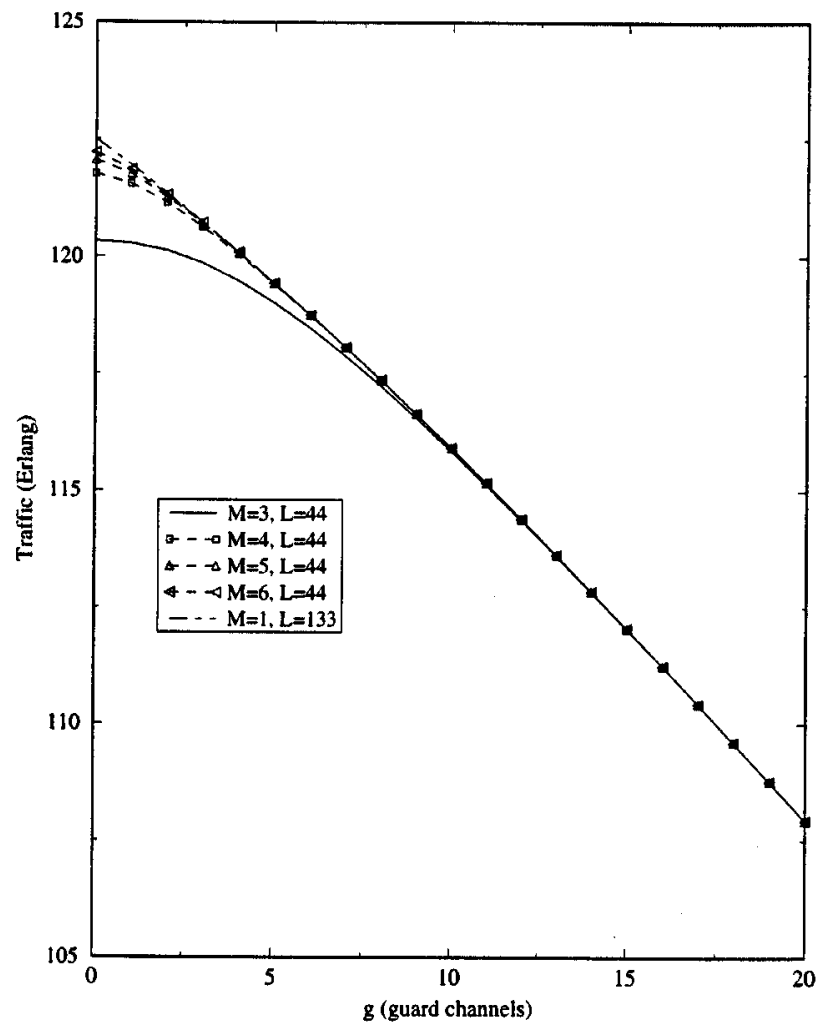

Fig. 14. Total carried traffic $C$ for different number of antenna elements $M$ with $L=44, \mathrm{SNR}=20, \gamma=18 \mathrm{~dB}, a=130, c=8$ (Erlangs) in a three-beam adaptive array system.

An important consequence of deploying adaptive arrays at the base station is the significant improvement in the total carried traffic (actual traffic that goes through) in the system. It means that a network with an adaptive array can effectively handle higher offered traffic intensities $a$, compared to the network with single omnidirectional antenna. The carried traffic is of great importance for most communication systems because blocked calls basically waste the network resources. Higher carried traffic means a smaller number of blocked calls. It is therefore highly desirable to maintain high total carried traffic in the network. The total carried traffic in the system is

$$
C=(a-c)\left(1-B_{O}\right)+c\left(1-B_{H}\right)
$$

where $C$ is the total carried traffic in the system, $a$ is the total offered traffic, and $c$ is the total handoff traffic in the system. Fig. 14 illustrates the effect of adaptive array on the total carried traffic in the system. In this example, it is assumed that there are $L=44$ channels available in the system. The total offered traffic $a$ is 130 Erlangs, and the total handoff traffic $c$ is 8 Erlangs. The total carried traffic for a different number of antenna elements $M$ in a three-beam system is plotted in Fig. 14. Fig. 14 confirms that a network with a three-beam adaptive array almost triples the capacity compared to network with an omnidirectional antenna and the same number of physical channels.

We also compare the average delay of handoff calls that actually do experience a delay, $D_{H}$ and $D_{\mathrm{QH}}$ (with $Q=1$ ), given by (41) and (53) respectively. The parameters here are again: $\mu=.012 / s, c=8$ Erlangs, and $L=44$. The average delay $D_{H}$ (infinite queue size) for two cases $K=1$ and $K=2$ are

$$
\begin{array}{ll}
D_{H}=2.31 \mathrm{~s} & \text { for single antenna }(K=1) \\
D_{H}=1.04 \mathrm{~s} & \text { for two-beam adaptive array }(K=2) .
\end{array}
$$

The average delay $D_{\mathrm{QH}}$ (finite queue size $Q=1$ ) for two cases $K=1$ and $K=2$ are

$$
\begin{array}{ll}
D_{1} H=1.89 \mathrm{~s} & \text { for single antenna }(K=1) \\
D_{1} H=0.95 \mathrm{~s} & \text { for 2-beam adaptive array }(K=2) .
\end{array}
$$

In any case, we see that from the traffic characteristic point of view, the network with an adaptive array outperforms the network with a single omnidirectional antenna.

\section{CONCLUSION}

A wireless communication network with beamforming capabilities at the base station is considered, and the blocking probabilities of the calls in the system under different traffic policies for such a network are derived. A novel approach is introduced that combines the effects of the digital signal processing (adaptive beamforming) at the physical layer and the traffic policies at the network layer on the overall queuing model of a cell. The effect of signal processing on the queuing model of the cell is represented by a parameter in the final cell model. The usefulness of adaptive arrays for improving the traffic characteristics of the network is confirmed through analytical as well as numerical results. Since wireless networks are witnessing a huge growth and becoming very popular, therefore adding new physical communication channels to the system becomes infeasible in most cases. The result of our analysis confirms that wireless networks with adaptive arrays are very promising in terms of traffic improvements in the network.

\section{REFERENCES}

[1] D. Everitt, "Traffic engineering of the radio interface for cellular mobile networks," Proc. IEEE, vol. 82, pp. 1371-1382, Sept. 1994.

[2] B. Jabbari, G. Colombo, and A. Nakajima, "Network issues for wireless communications," in IEEE Commun. Mag., Jan. 1995, pp. 88-102.

[3] D. Everitt, "Combined analysis of transmission and traffic in micro cellular mobile communications system," in Proc. IEEE Vehicular Technology Conf., May 1993, pp. 577-580.

[4] E. Posner and R. Guerin, "Traffic policies in cellular radio that minimizes blocking of handoff calls," in Proc. IEEE Int. Teletraffic Conf., Japan, Sept. 1985, pp. 249-298.

[5] R. Guerin, "Queueing-blocking system with two arrivals stream and guard channels," IEEE Trans. Commun., vol. 36, pp. 153-163, Feb. 1988.

[6] J. Razavilar, "Signal processing and performance analysis for optimal resource allocation in wireless networks," Ph.D. dissertation, Univ. of Maryland, 1998.

[7] J. Razavilar, F. Rashid-Farrokhi, and K. J. R. Liu, "Joint beamforming and handoff for traffic improvements in wireless networks," in Proc. IEEE Int. Conf. Communications (ICC'99), vol. 1, June 1999, pp. 41-45.

[8] J. Ward and R. T. Compton, "High throughput slotted aloha packet radio networks with adaptive arrrays," in Proc. IEEE Int. Conf. Communications, Mar. 1993, pp. 460-470.

[9] J. H. Winters, "Smart antennas for wireless systems," IEEE Personal Commun. Mag., vol. 5, pp. 23-27, Feb. 1998.

[10] R. A. Monzingo and T. W. Miller, Introduction to Adaptive Arrays. New York: Wiley, 1980. 
[11] S. Haykin, Adaptive Filter Theory. Englewood Cliffs, NJ: PrenticeHall, 1991.

[12] A. Naguib, A. Paulraj, and T. Kailath, "Capacity improvement with basestation antenna arrays in cellular cdma," IEEE Trans. Veh. Technol., vol. 34, pp. 691-698, Aug. 1994.

[13] B. Suard, A. Naguib, G. Xu, and A. Paulraj, "Performance analysis of cdma mobile communication systems using antenna array," in Proc. IEEE Int. Conf. Acoustics, Speech and Signal Processing, Apr. 1993, pp. $153-156$.

[14] G. Xu, Y. Cho, A. Paulraj, and T. Kailath, "Maximum likelihood detection of co-channel communication signals via exploitation of spatial diversity," in Proc. 26th Asilomar Conf. Signals, Systems, and Computers, Pacific Grove, CA, Oct. 1992, pp. 1142-1146.

[15] A. Klouche-Djedid and M. Fujita, "Adaptive array processing applications for mobile telephone communication," IEEE Trans. Veh. Technol., pp. 405-416, Aug. 1996.

[16] S. Swales, M. Beach, D. Edwards, and J. McGeehan, "The performance enhancement of multibeam adaptive base-station antenna for cellular land mobile radio systems," IEEE Trans. Veh. Technol., pp. 56-67, Feb. 1990.

[17] T. Gebauer and H. Gockler, "Channel-individual adaptive beamforming for mobile satellite communications," IEEE J. Select. Areas Commun., pp. 439-448, Feb. 1995.

[18] S. J. Orfanidis, Optimal Signal Processing: An Introduction. New York: Macmillan, 1985

[19] R. Cooper, Introduction to Queueing Theory. New York: North-Holland, 1981.

[20] L. Kleinrock, Queueing Systems. New York: Wiley, 1985.

[21] J. Razavilar, F. Rashid-Farrokhi, and K. Liu, "Blocking probability of handoff calls carried traffic in wireless networks with antenna arrays," in Proc. 31st Asilomar Conf. Signals, Systems, and Computers, Pacific Grove, CA, Nov. 1997, pp. 635-639.

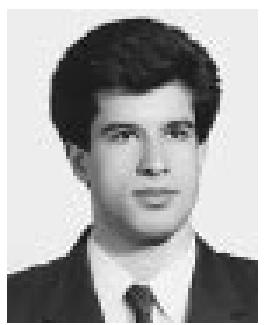

Javad Razavilar (S'89-M'99) received the B.S. degree from Sharif University of Technology, Tehran, Iran, in 1989 and the M.S. and Ph.D. degrees from the University of Maryland at College Park in 1996 and 1998, respectively, both in electrical engineering.

He joined the Technology Development Center, 3COM Corp., San Diego, CA, as a Senior Member of Technical Staff in 1999. From 1994 to 1998, he was a Research Assistant at the Institute for Systems Research (ISR), University of Maryland. From 1993 to 1994, he was a Teaching Assistant in the Electrical Engineering Department of the University of Maryland. His research interests are stochastic optimization, statistical signal processing, digital communications, information theory, networking, and wireless networks.

Dr. Razavilar is a member of Eta Kappa Nu, Gamma Xi chapter. He is also a member of the IEEE Information Theory, Communications, and Signal Processing societies.

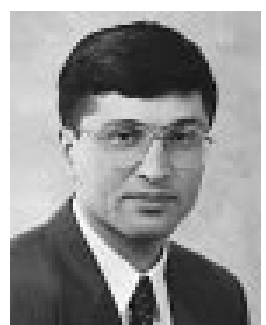

Farrokh Rashid-Farrokhi (S'88-M'97) received the B.S. and M.S. degrees (highest honors) in electrical engineering from Sharif University of Technology, Tehran, Iran, in 1988 and 1992, respectively, and the $\mathrm{Ph} . \mathrm{D}$. degree in electrical engineering from the University of Maryland at College Park.

He joined the Wireless Communications Research Department, Bell Laboratories, Holmdel, NJ, as a Member of Technical Staff in 1998. His research interests include array and statistical signal processing, wireless communications, and networking.

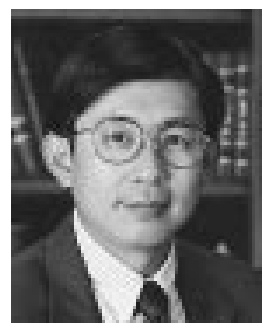

K. J. Ray Liu (S'86-M'90-SM'93) received the B.S. degree from the National Taiwan University, Taiwan, R.O.C., in 1983 and the Ph.D. degree from the University of California, Los Angeles, in 1990, both in electrical engineering.

Since 1990, he has been with the Electrical Engineering Department and Institute for Systems Research, University of Maryland at College Park, where he is an Associate Professor. During his sabbatical leave in 1996-1997, he was a Visiting Associate Professor at Stanford University, Stanford, CA. His research interests span various aspects of signal/image processing and communications. He has published more than 160 papers, of which more than 60 are in archival journals and book chapters. He is an Editor of the Journal of VLSI Signal Processing Systems and the Series Editor of a Marcel Dekker series on signal processing

Dr. Liu has received numerous awards including the 1994 National Science Foundation Young Investigator Award, the IEEE Signal Processing Society's 1993 Senior Award (Best Paper Award), the IEEE Benelux Joint Chapter on Vehicular Technology and Communications 1999 Award, the George Corcoran Award in 1994 for outstanding contributions to electrical engineering education, the 1995-96 Outstanding Systems Engineering Faculty Award in recognition of outstanding contributions in interdisciplinary research, both from the University of Maryland, and many others. Dr. Liu was an Associate Editor of the IEEE TRANSACTIONS ON Signal Processing, a Guest Editor of special issues on multimedia signal processing of PROCEEDINGS OF THE IEEE, and a Guest Editor of a special issue on signal processing for wireless communications of the IEEE JOURNAL OF SELECTED AREAS IN COMMUNICATIONS. He currently is Chair of the Multimedia Signal Processing Technical Committee of the IEEE Signal Processing Society, a Guest Editor of a special issue on multimedia over IP of the IEEE TRANSACTIONS ON MULTIMEDIA, and a Guest Editor of a special issue on robust video transmission of the IEEE SIGNAL PROCESSING MAGAZINE. 